Supplement of The Cryosphere, 13, 2817-2834, 2019

https://doi.org/10.5194/tc-13-2817-2019-supplement

(C) Author(s) 2019. This work is distributed under

the Creative Commons Attribution 4.0 License.

(c) (i)

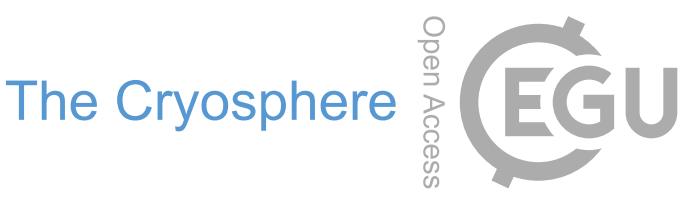

Supplement of

\title{
Melt at grounding line controls observed and future retreat of Smith, Pope, and Kohler glaciers
}

David A. Lilien et al.

Correspondence to: David A. Lilien (dal22@uw.edu)

The copyright of individual parts of the supplement might differ from the CC BY 4.0 License. 


\section{Model initialization}

The modeled ice temperature depends on the inferred basal shear stress and ice viscosity because of their effect on strain heating within the ice and frictional heating at the bed, and the inferred velocity field depends on the temperature because the rheology of ice depends on its temperature. This coupling thus necessitates recursive updating of the estimates of these fields to reach a consistent solution that matches the observed velocity.

\subsection{Diagnostic temperature model}

Since vertical variations in temperature are generally larger than horizontal variations, for both shallow-shelf and fullStokes models it is necessary to determine the temperature in three dimensions. Thus, to initialize both shallow-shelf and full-Stokes models, we used a full-Stokes diagnostic model to determine the temperature. The model solves the advection-diffusion equations for heat within the ice, accounting for heat production from internal deformation and from basal friction (Zwinger et al., 2007). The upper surface was fixed to the 1979-2013 mean temperature from RACMO 2.3 (Van Wessem et al., 2014), and the upstream boundaries used a simple ice-divide temperature profile (Cuffey \& Paterson, 2010). Over grounded ice, we imposed an incoming flux equal to the geothermal heat on the lower surface (Maule et al., 2005), while over floating ice we assumed that the temperature was equal to the pressure melting point. After finding the temperature field, we extracted the depth-averaged temperature and flow coefficient $(B)$ for use with the shallow-shelf model and used the inferred temperature field directly with the full-Stokes model.

\subsection{Inversion procedure}

We separately determined the basal shear stress beneath grounded ice and the depth-averaged enhancement factor over floating ice that minimized the misfit between modelled velocity and InSAR observations from 1996. Leaving a single free parameter in each area of the domain prevented overfitting. For both inversion procedures, adjoint methods were used to determine the parameter values that caused the best fit with observations (MacAyeal, 1993; Morlighem et al., 2010). We did not regularize the enhancement factor inversions, while for the basal shear stress we used an Lcurve (Calvetti et al., 2000) to select the regularization coefficient. Though the methods were identical, this procedure was performed separately for shallow-shelf and full-Stokes models because the basal shear stress in particular is fundamentally different between the two models; because all motion is accommodated by sliding in the shallow-shelf model, basal resistance must generally be inferred to be lower in order to allow flow to be as fast as observations. The friction coefficient corresponding to the Schoof (2005) sliding law was computed from the basal shear stress and basal velocity fields, and then the friction coefficient and depth-averaged enhancement factor were used as inputs for the iterative determination of the temperature field and subsequently for initialization of the forward model.

\subsection{Relaxation}

In order to prevent spurious, transient effects caused by erroneous data from affecting our analysis, we allowed the model geometry to evolve for one year, using the full-Stokes model, then used the resulting geometry to re-determine 
the temperature and inferred parameters. In the context of ongoing changes, relaxation is necessarily a compromise between trying to capture real transient effects and removing artificial effects resulting from data or model errors. For example, because sub-shelf melt exceeded grounding-line flux which in turn exceeded basin-wide accumulation, any melt forcing will necessarily be out of balance with either grounded- or floating-ice loss. During the relaxation, the model was forced using an estimate of the melt in 1996 (Lilien et al., 2018), leading to slight (GT HERE) mass loss during the relaxation process. Once the new geometry was obtained, we again iterated between temperature modelling and inverse modelling (with both the full-Stokes and shallow-Shelf models) to best match the velocities in 1996. These final inferred values, as described above, were then used as the initial conditions for the longer prognostic simulations.

\section{Model limitations}

Here we show that the central modeling results are not limited by the mesh resolution or the sliding law.

\subsection{Model resolution}

We ran simulations using both the full-Stokes and shallow-shelf models at three resolutions to determine whether the meshes used were sufficiently fine to accurately capture grounding-line dynamics. We used $300 \mathrm{~m}, 500 \mathrm{~m}$, and 1000 $\mathrm{m}$ horizontal grounding-line resolution, with resolution away from the grounding line ten times coarser in all cases. These meshes had 3, 7, and 9 vertical layers, respectively, for the full-Stokes runs. The evolution of the grounding line for each of these resolutions is shown in Figure S1. We find that the mesh resolution affects the timing of retreat, the change is only $\sim 10$ years for the shallow shelf model and $\sim 5$ years for the full-Stokes model. However, whether retreat occurs, and the rate at which it occurs once initiated, is similar regardless of mesh resolution. In light of this similarity, and past studies that have indicated that $300 \mathrm{~m}$ is sufficient resolution near the grounding line (Pattyn et al., 2013; Seroussi \& Morlighem, 2018; Sun et al., 2016), we consider the $300 \mathrm{~m}$ runs sufficiently resolved for meaningful interpretation.
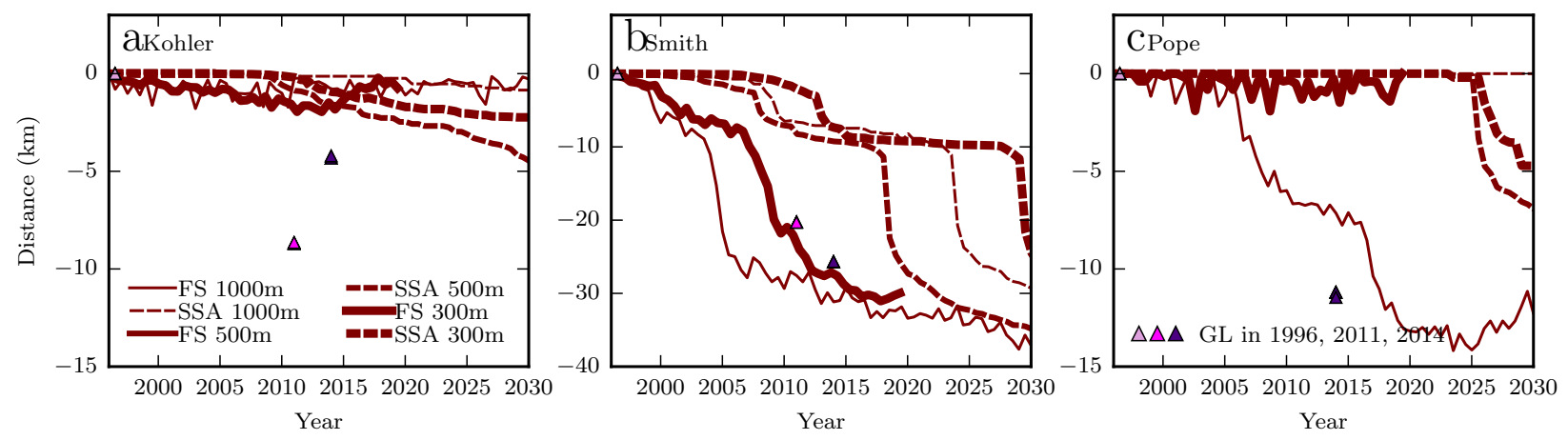

Figure S1. Sensitivity of grounding line position to mesh resolution. a-c. Position of grounding line through time along transects of Kohler, Smith, and Pope centerlines, respectively, from Figure 1 of the main text. Line style indicates model physics: solid for full Stokes and dashed for shallow shelf. Line thickness indicates mesh resolution near the grounding line, with thicker lines indicating higher resolution. Purple triangles indicate observed grounding-line positions through time. 


\subsection{Sliding law}

All the simulations discussed in the main text were conducted using a Coulomb-type sliding law (Gagliardini et al., 2007; Schoof, 2005). For comparison, we ran set of four simulations using the shallow-shelf model, each with different melt forcing at 1 Obs intensity, using a Weertman sliding law with $m=3$. The rate of grounding line retreat is relatively insensitive to using this other sliding law, though some differences in timing are observed (Figure S2). We do not further interpret the differences between these runs, but simply note that our primary conclusions about what forcings result in grounding-line retreat are not sensitive to the choice between these commonly used sliding laws. However, we consider the coulomb-type sliding law to be the best choice for such simulations given present knowledge about the sliding process; multiple studies have found nearly plastic behavior underneath fast-moving ice and Weertman sliding with smaller exponents in slower moving regions (Gillet-Chaulet et al., 2016; Joughin et al., 2009).

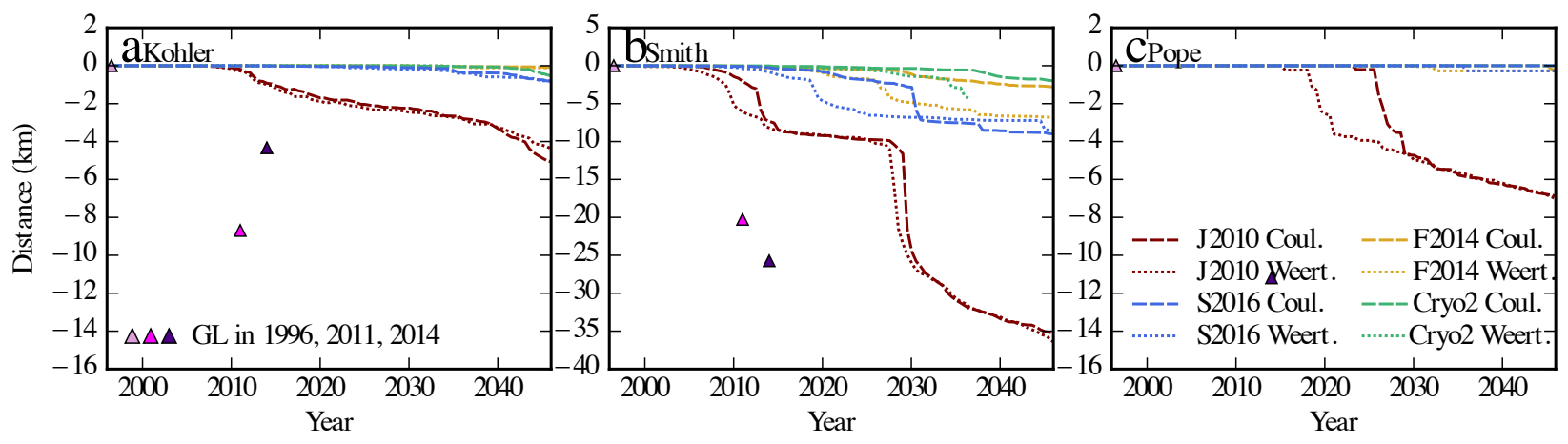

Figure S2. Sensitivity of grounding-line position to sliding law. a-c. Position of grounding line through time along transects of Kohler, Smith, and Pope centerlines, respectively, from Figure 1 of the main text. Line style indicates sliding law, with the Schoof (2005) sliding law that was used in the main text as the dashed lines and a Weertman sliding law with exponent $\boldsymbol{m}=\mathbf{3}$ as the dotted line. Color indicates melt forcing used. Purple triangles indicate observed grounding-line positions through time. 